\title{
锌-锂双金属化合物的合成及其在异腈酸酯硼氢化中的催化应用
}

\author{
肖铃 $a$ 㶓沈带 $a$ 陈泽卫 ${ }^{b}$ 姚薇薇*,b 郑 晶*,a 马猛涛*,a \\ ( ${ }^{a}$ 南京林业大学理学院 南京 210037) \\ ( ${ }^{b}$ 南京中医药大学药学院 南京 210023)
}

\begin{abstract}
摘要 有关异腈酸酯嗍氢化反应的报道极其稀少，迄今为止，锌金属化合物催化的异腈酸酯硼氢化反应尚未见报道. 介绍了一种结构新颖的不对称 $\beta$-二亚胺锌-锂双金属化合物的合成方法，并将其作为高效催化剂成功应用于一系列带 有不同官能团的异腈酸酯和频哪醇嗍烷的嗍氢化反应中, 同时对硼氢化反应机理进行了初步探索.

关键词 锌一锂双金属; 异腈酸酯; 硼氢化; 合成
\end{abstract}

\section{Synthesis of Zn-Li Bimetallic Compound and Its Catalytic Application in Hydroboration of Isocyanate}

\author{
Xiao, Qian $^{a} \quad$ Zang, Shenluo $^{a} \quad$ Chen, Zewei $^{b} \quad$ Yao, Weiwei $^{*, b}$ \\ Zheng, Jing ${ }^{*, a} \quad$ Ma, Mengtao ${ }^{*, a}$ \\ $\left({ }^{a}\right.$ College of Science, Nanjing Forestry University, Nanjing 210037) \\ $\left({ }^{b}\right.$ College of Pharmacy, Nanjing University of Chinese Medicine, Nanjing 210023)
}

\begin{abstract}
There are very few reports on the isocyanate hydroboration. So far, the $\mathrm{Zn}$-catalyzed hydroboration of isocyanate has not been reported. The synthesis of a novel asymmetric $\beta$-diketiminate $\mathrm{Zn}$-Li bimetallic compound and its application as a highly efficient catalyst in the hydroboration of various isocyanates with HBpin in high yields are reported. The preliminary mechanism of hydroboration reaction has been explored.
\end{abstract}

Keywords Zn-Li bimetallic compound; isocyanate; hydroboration; synthesis

锌是人体必需的微量元素之一, 正常的氧化态是 +2 价. 一价录很早就被人们熟知 ${ }^{[1]}$, 然而同族的一价 锌化合物直到 2004 年才被西班牙的 Carmona 等人在一 次偶然的实验中发现, 他们用五甲基环戊二烯基二价锌 化合物 $\left[\mathrm{Zn}\left(\mathrm{Cp}^{*}\right)_{2}\right]$ 和二乙基锌 $\left(\mathrm{ZnEt}_{2}\right)$ 反应意外地得到了 稳定一价锌化合物 $\left[\mathrm{Zn}_{2}\left(\eta^{5}-\mathrm{Cp}^{*}\right)_{2}\right]$, 然后用 $\left[\mathrm{Zn}\left(\mathrm{Cp}^{*}\right)_{2}\right]$, $\mathrm{ZnCl}_{2}$ 和 $\mathrm{KH}$ 以 $1: 1: 2$ 的物质的量比以更方便的方法 高产率得到相同的一价锌化合物 $\left[\mathrm{Zn}_{2}\left(\eta^{5}-\mathrm{Cp}^{*}\right)_{2}\right]^{[2-3]}$. 这 一突破性的发现立刻引起了其他研究人员浓厚的兴趣, 很快就用各种不同的配体合成出相应的一价锌化合 物 ${ }^{[4-7]}$. 但通过对文献的仔细检索, 发现一价锌化合物作 为催化剂应用于催化反应的报道极其稀少 ${ }^{[8]}$. 迄今为止, 唯一的例子是 Roesky 等发现在 $2.5 \mathrm{~mol} \%$ 助催化剂
$\left[\mathrm{PhNMe}_{2} \mathrm{H}\right]\left[\mathrm{B}\left(\mathrm{C}_{6} \mathrm{~F}_{5}\right)_{4}\right]$ 的共同作用下 $2.5 \mathrm{~mol} \%\left[\mathrm{Zn}_{2}\left(\eta^{5}-\right.\right.$ $\left.\mathrm{Cp}^{*}\right)_{2}$ ] 能高效催化苯胺和炔的氢氨化反应 ${ }^{[9-10]}$.

近几年来, 不饱和有机底物嗍氢化受到广泛的关注 和研究 ${ }^{[11-21]}$, 然而对于异腈酸酯嗍氢化反应的报道极其 稀少. 2016 年 Warren 和 Grimme 等 ${ }^{[22]}$ 通过大空间位阻异 腈酸酯直接与 Piers 硼烷 $\mathrm{HB}\left(\mathrm{C}_{6} \mathrm{~F}_{5}\right)_{2}$ 的硼氢化反应合成出 一类新的 “受阻路易斯酸碱对” (frustrated Lewis Pairs, FLPs). 同年, Okuda 及其合作者 ${ }^{[23]}$ 使用镁金属化合物 $\left[\mathrm{Mg}(\mathrm{THF})_{6}\right]\left[\mathrm{HBPh}_{3}\right]_{2}$ 作为催化剂, 将叔丁基异腈酸酯与 频哪醇硼烷(HBpin)以 $1: 2$ 的物质的量比反应, 得到了 二硼氢化的亚甲基胺硼化物(Scheme 1A). 2017 年, Fang 和 Hill 等 ${ }^{[24]}$ 将镁丁基化物 $\left[\left(^{\mathrm{Dipp}} \mathrm{Nacnac}\right) \mathrm{Mg}^{n} \mathrm{Bu}\right]$ 作为催化 剂应用于异腈酸酯和频哪醇硼烷的硼氢化反应中, 当异

\footnotetext{
* Corresponding authors. E-mail: yww0715@hotmail.com,jzheng62@njfu.edu.cn,mengtao@njfu.edu.cn Received June 15, 2020; revised July 26, 2020; published online August 18, 2020.

Project supported by the National Natural Science Foundation of China (Nos. 21772093, 21372117), the Natural Science Foundation of Jiangsu Province (No. BK20181421) and the Postgraduate Research \& Practice Innovation Program of Jiangsu Province (No. KYCX18_0983).

国家自然科学基金(Nos. 21772093, 21372117)、江苏省自然科学基金(No. BK20181421)和江苏省研究生科研与实践创新计划(No. KYCX18_0983)资助 项目.
} 
腈酸酯底物与 HBpin 的物质的量比为 $1: 3$ 时, 可得到 完全硼氢化的甲基胺硼化物(Scheme 1B). 甲基胺硼化 物可以作为有效的氨基化试剂, 如 Fernández 等 ${ }^{[25]}$ 使用 烷基胺硼化物与 $\alpha, \beta$-不饱和羰基化合物反应, 生成 $\beta$-氨 基羰基化合物. 令人惊奇的是, 迄今为止, 除了上述镁 金属化合物催化异腈酸酯硼氢化反应，未有其他金属化 合物催化异腈酸酯硼氢化的报道. 最近在制备不对称一 价锌化合物的过程中, 成功合成出一种结构新颖的不对 称 $\beta$-二亚胺配位的锌-锂双金属化合物 2 , 并尝试了其 在异腈酸酯硣氢化反应中的催化应用, 发现其作为催化 剂高效催化一系列异腈酸酯的硼氢化反应, 可在较短时 间内获得更高产率, 且具有良好的官能团耐受性, 并对 反应机理进行了初步探索(Scheme 1C).

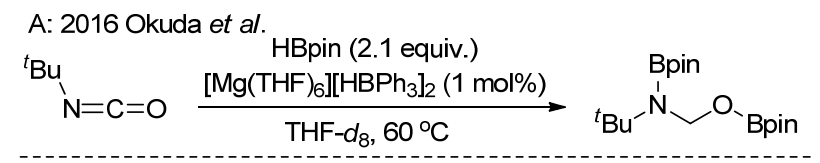

B: 2017 Fang and Hill et al.

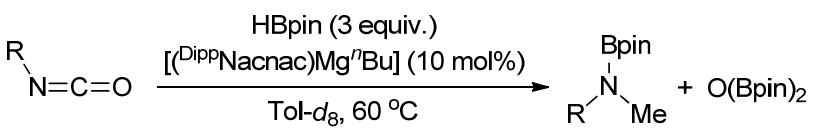

C: This work

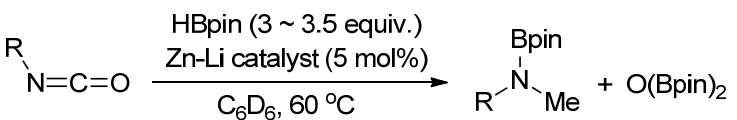

图式 1 异腈酸脂的嗍氢化反应

Scheme 1 Hydroboration of isocyanate

\section{2 结果与讨论}

\section{1 锌化合物的合成及晶体结构分析}

近期我们成功合成了一系列不对称 $\beta$-二亚胺配体 稳定的一价镁金属化合物 ${ }^{[26]}$, 因此猜想这类配体能否 应用于不对称一价锌化合物的合成. 在对甲苯磺酸的催 化下，2,6-二异丙基苯胺 $\left(\mathrm{DippNH}_{2}\right)$ 先与 2,4-戊二酮以 $1: 1$ 的物质的量比反应得到单取代烯酮中间体, 然后 再与 2,4,6-三甲基苯胺 $\left(\mathrm{MesNH}_{2}\right)$ 反应制备出相应的不对 称 $\beta$-二亚胺配体 1 . 低温下配体 1 与正丁基锂反应, 去 质子化得到相应的配体锂盐, 然后再与碘化锌的乙醚溶 液继续反应, 分离得到不对称 $\beta$-二亚胺锌锂双金属碘化 物 2. 通过 $X$ 射线单晶衍射表征, 确定其以锌锂双金属 桥连碘化物为核心的空间结构, 其中锂原子上有两个配 位的乙醚分子以及两个桥连的碘原子. 化合物 2 的核磁 共振数据也与目标产物一致. 最后我们将不对称 $\beta$-二亚 胺锌的碘化物用过量金属钠进行还原, 希望得到不对称 $\beta$-二亚胺稳定的一价锌金属化合物. 即使在氩气保护 下, 在处理的过程中也不断有黑色粉末析出, 我们推测 其应为不对称一价锌化合物, 由于反应活性太活泼而发
生分解析出金属锌单质.同时我们也得到一种无色晶 体，对该晶体进行 X 射线衍射表征，发现其结构并非预 期的一价锌金属化合物，而是二聚体锌化合物 3. 这可 能是由于目标产物的稳定性较差导致常温下一价锌化 合物发生歧化反应分解生成二价锌化合物和锌金属单 质(Scheme 2).
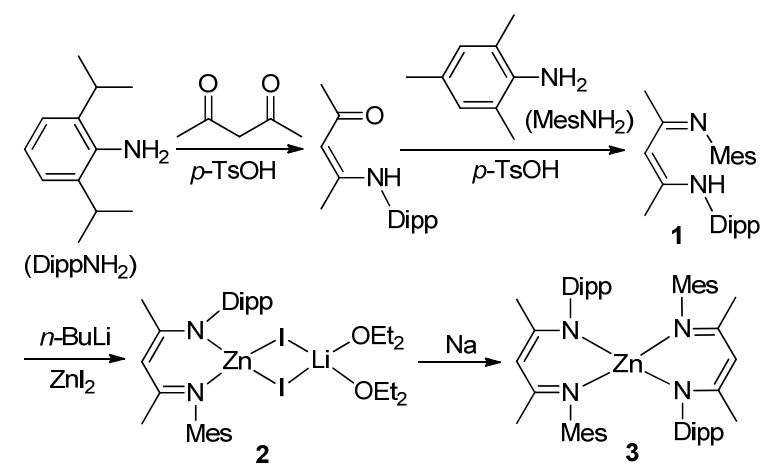

图式 2 锌金属化合物的合成

Scheme 2 Syntheses of $\mathrm{Zn}$ compounds

化合物 2 和 3 晶体结构如图 1 和图 2 所示, 相应的 主要键长和键角见表 1 和表 2 , 详细的晶体参数见表 3 . 如图 1 所示, 晶体结构显示化合物 2 为 $\mathrm{Zn}-\mathrm{Li}$ 双金属化 合物, 锌原子和锂原子通过 2 个碘原子相互桥连, 形成 略微扭曲的平面四边形, 锌原子通过与 $\beta$-二亚胺配体的 2 个氮原子配位形成一个略微扭曲的六元环. 四配位的 锌原子为扭曲的四面体构型, $\beta$-二亚胺配体的 2 个氮原 子和桥连的 2 个碘原子分别占据四面体的四个顶点. 锂 原子同样也为扭曲的四面体构型，四个顶点分别为乙醚 的 2 个氧原子和桥连的 2 个碘原子所占据. $\mathrm{Zn}-\mathrm{N}$ 键长 几乎等长(0.1976/0.1979 $\mathrm{nm}), \mathrm{Zn}(1)-\mathrm{I}(2)$ 比 $\mathrm{Zn}(1)-\mathrm{I}(1)$ 略长 $(0.2660 / 0.2592 \mathrm{~nm})$, 而 $\mathrm{Li}(1)-\mathrm{I}(2)$ 与 $\mathrm{Li}(1)-\mathrm{I}(1)$ 相 比则稍短 $(0.2800 / 0.2872 \mathrm{~nm})$, 这处于对称配体锌锂双金 属化合物 $\mathrm{Li}-\mathrm{I}$ 的键长之间 $(0.2784 / 0.2919 \mathrm{~nm})^{[27]}$.

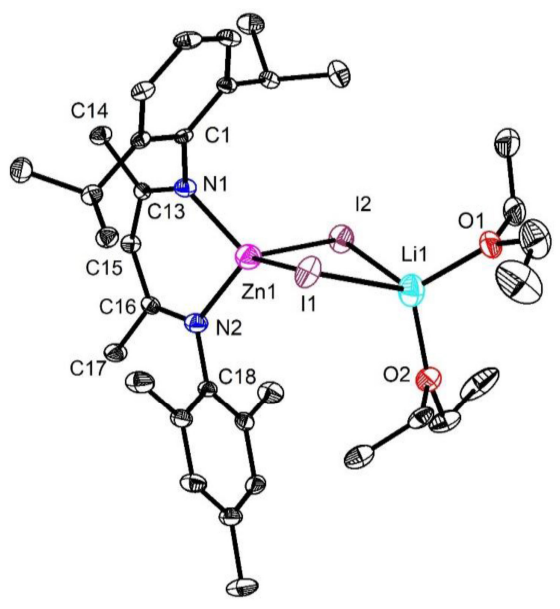

图 1 化合物 2 的晶体结构

Figure 1 Crystal structure of compound 2 


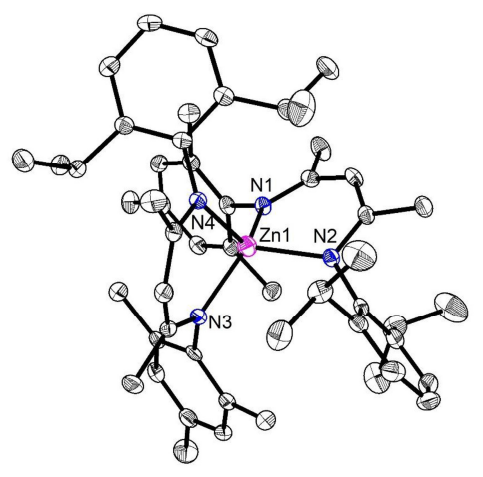

图 2 化合物 $\mathbf{3}$ 的晶体结构

Figure 2 Crystal structure of compound 3

表 1 化合物 2 的部分键长 $(\mathrm{nm})$ 和键角 $\left(^{\circ}\right)$

Table 1 Selected bond lengths (nm) and bond angles $\left(^{\circ}\right.$ ) for compound 2

\begin{tabular}{llll}
\hline $\mathrm{Zn}(1)-\mathrm{N}(1)$ & $0.1976(2)$ & $\mathrm{Zn}(1)-\mathrm{N}(2)$ & $0.1979(3)$ \\
$\mathrm{Zn}(1)-\mathrm{I}(1)$ & $0.2592(4)$ & $\mathrm{Zn}(1)-\mathrm{I}(2)$ & $0.2660(4)$ \\
$\mathrm{Li}(1)-\mathrm{I}(1)$ & $0.2872(6)$ & $\mathrm{Li}(1)-\mathrm{I}(2)$ & $0.2800(6)$ \\
$\mathrm{Li}(1)-\mathrm{O}(1)$ & $0.1927(7)$ & $\mathrm{Li}(1)-\mathrm{O}(2)$ & $0.1910(7)$ \\
$\mathrm{N}(1)-\mathrm{Zn}(1)-\mathrm{N}(2)$ & $98.21(11)$ & $\mathrm{I}(1)-\mathrm{Zn}(1)-\mathrm{I}(2)$ & $102.42(15)$ \\
$\mathrm{N}(1)-\mathrm{Zn}(1)-\mathrm{I}(1)$ & $115.43(7)$ & $\mathrm{N}(1)-\mathrm{Zn}(1)-\mathrm{I}(2)$ & $112.78(7)$ \\
$\mathrm{N}(2)-\mathrm{Zn}(1)-\mathrm{I}(1)$ & $122.50(7)$ & $\mathrm{N}(2)-\mathrm{Zn}(1)-\mathrm{I}(2)$ & $105.50(7)$ \\
$\mathrm{Zn}(1)-\mathrm{I}(1)-\mathrm{Li}(1)$ & $80.33(13)$ & $\mathrm{Zn}(1)-\mathrm{I}(2)-\mathrm{Li}(1)$ & $80.53(12)$ \\
$\mathrm{I}(1)-\mathrm{Li}(1)-\mathrm{I}(2)$ & $92.39(18)$ & $\mathrm{O}(1)-\mathrm{Li}(1)-\mathrm{O}(2)$ & $109.40(3)$ \\
$\mathrm{I}(1)-\mathrm{Li}(1)-\mathrm{O}(1)$ & $121.90(3)$ & $\mathrm{I}(1)-\mathrm{Li}(1)-\mathrm{O}(2)$ & $108.20(3)$ \\
$\mathrm{I}(2)-\mathrm{Li}(1)-\mathrm{O}(1)$ & $108.20(3)$ & $\mathrm{I}(2)-\mathrm{Li}(1)-\mathrm{O}(2)$ & $116.40(3)$ \\
\hline
\end{tabular}

表 2 化合物 3 的部分键长 $(\mathrm{nm})$ 和键角 $\left(^{\circ}\right)$

Table 2 Selected bond lengths (nm) and bond angles $\left(^{\circ}\right)$ for compound $\mathbf{3}$

\begin{tabular}{llll}
\hline $\mathrm{Zn}(1)-\mathrm{N}(1)$ & $0.2031(18) \mathrm{Zn}(1)-\mathrm{N}(2)$ & $0.2033(17)$ \\
$\mathrm{Zn}(1)-\mathrm{N}(3)$ & $0.2017(17) \mathrm{Zn}(1)-\mathrm{N}(4)$ & $0.2051(17)$ \\
\hline $\mathrm{N}(1)-\mathrm{Zn}(1)-\mathrm{N}(2)$ & $94.71(7)$ & $\mathrm{N}(1)-\mathrm{Zn}(1)-\mathrm{N}(3)$ & $113.49(7)$ \\
$\mathrm{N}(1)-\mathrm{Zn}(1)-\mathrm{N}(4)$ & $114.80(7)$ & $\mathrm{N}(2)-\mathrm{Zn}(1)-\mathrm{N}(3)$ & $124.91(7)$ \\
$\mathrm{N}(2)-\mathrm{Zn}(1)-\mathrm{N}(4)$ & $115.96(7)$ & $\mathrm{N}(3)-\mathrm{Zn}(1)-\mathrm{N}(4)$ & $94.43(7)$ \\
\hline
\end{tabular}

如图 2 所示, 晶体结构表征显示化合物 3 中锌原子 被两个 $\beta$-二亚胺配体包围, 锌与两个配体上的四个氮原 子配位形成略微扭曲的四面体构型. $\mathrm{Zn}-\mathrm{N}$ 键长 $[0.2017(17) \sim 0.2051(17) \mathrm{nm}]$ 比 2 中相应键长 $[0.1976(2)$, $0.1979(3) \mathrm{nm}$ ]较长, 两个 $\mathrm{C}_{3} \mathrm{~N}_{2} \mathrm{Zn}$ 六元环几乎相互垂直.

\section{2 锌-锂双金属化合物在异腈酸酯磞氢化中的催化 应用及反应机理研究}

首先在无催化剂的条件下, 我们将异丙基异腈酸酯 和频哪醇嗍烷以 $1: 3$ 的物质的量比在氝代苯中进行空 白反应, 即使加热到 $60{ }^{\circ} \mathrm{C}$ 反应 $12 \mathrm{~h}$, 也没有观察到硼 氢化产物的生成(Table 4, Entries 1, 2). 然而在上述混合 物中加入 $5 \mathrm{~mol} \% 2$ 作为催化剂, 室温下反应 $24 \mathrm{~h}$ 得到 $23 \%$ 产率. 升高反应温度至 $60{ }^{\circ} \mathrm{C}, 12 \mathrm{~h}$ 后即可获得 $93 \%$ 产率(Table 4, Entries 3, 4). 增加催化剂量至 $10 \mathrm{~mol} \%$,
表 3 化合物 $\mathbf{2}$ 和 $\mathbf{3}$ 的晶体参数

Table 3 Crystallographic data for compounds 2 and 3

\begin{tabular}{lll}
\hline Compd. & \multicolumn{1}{c}{$\mathbf{2}$} & \multicolumn{1}{c}{$\mathbf{3}$} \\
\hline Formula & $\mathrm{C}_{34} \mathrm{H}_{55} \mathrm{I}_{2} \mathrm{LiN}_{2} \mathrm{O}_{2} \mathrm{Zn}$ & $\mathrm{C}_{107} \mathrm{H}_{147} \mathrm{~N}_{8} \mathrm{Zn}_{2}$ \\
$M_{\mathrm{r}}$ & 849.91 & 1676.11 \\
Temp./K & $130(2)$ & $120(2)$ \\
$\lambda / \mathrm{nm}$ & 0.071073 & 0.071073 \\
Crystal system & Monoclinic & Monoclinic \\
Space group & $P{ }_{1} / c$ & $P 2_{1} / n$ \\
$a / \mathrm{nm}$ & $1.15779(9)$ & $1.08285(4)$ \\
$b / \mathrm{nm}$ & $3.0070(2)$ & $2.17902(8)$ \\
$c / \mathrm{nm}$ & $1.24889(9)$ & $4.01324(15)$ \\
$\alpha /\left(^{\circ}\right)$ & 90 & 90 \\
$\beta /\left(^{\circ}\right)$ & $116.076(2)$ & $95.7710(10)$ \\
$\gamma /\left({ }^{\circ}\right)$ & 90 & 90 \\
$V / \mathrm{nm}{ }^{3}$ & $3.9054(5)$ & $9.4215(6)$ \\
$Z$ & 4 & 4 \\
$\rho_{\text {calc }} /\left(\mathrm{g} \cdot \mathrm{cm}^{-3}\right)$ & 1.446 & 1.182 \\
$\mu / \mathrm{mm}^{-1}$ & 2.238 & 0.560 \\
$F(000)$ & 1712 & 3620 \\
$R_{1}($ obs. data $)$ & 0.0289 & 0.0384 \\
$w R_{2}($ obs. data $)$ & 0.0629 & 0.0866 \\
$\mathrm{GOF}$ on $F^{2}$ & 1.094 & 1.030 \\
$\mathrm{CCDC}^{-1}$ & 2008454 & 2008455 \\
\hline
\end{tabular}

表 4 异腈酸酯硼氢化反应条件的优化

Table 4 Optimization of isocyanate hydroboration

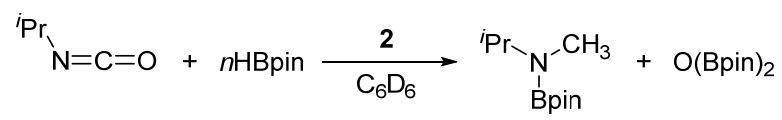

\begin{tabular}{cccccc}
\hline Entry & Cat. $/ \mathrm{mol} \%$ & $n$ & Temp. $/{ }^{\circ} \mathrm{C}$ & Time $/ \mathrm{h}$ & Yield $^{a} / \%$ \\
\hline 1 & 0 & 3 & 25 & 12 & 0 \\
2 & 0 & 3 & 60 & 12 & 0 \\
3 & 5 & 3 & 25 & 24 & 23 \\
4 & 5 & 3 & 60 & 12 & 93 \\
5 & 10 & 3 & 60 & 12 & 95 \\
6 & 5 & 3 & 60 & 6 & 70 \\
7 & 5 & 3.5 & 60 & 6 & 99 \\
\hline
\end{tabular}

${ }^{a}$ The yield was determined by ${ }^{1} \mathrm{H}$ NMR spectroscopy.

产率略有提升(Table 4, Entry 5). 减少反应时间至 $6 \mathrm{~h}$, 产率仅为 70\% (Table 4, Entry 6). 此后增加频哪醇嗍烷 的比例至 3.5, 可以在较短的反应时间内 $(6 \mathrm{~h})$ 使异丙基 异腈酸酯完全转化(Table 4, Entry 7).

在确定最佳的反应条件后, 我们对异腈酸酯底物进 行了拓展. 在 $5 \mathrm{~mol} \% 2$ 的催化下，一系列带有不同取代 基(乙基、正丙基、正丁基等)的脂肪族异腈酸酯与频哪 醇硼烷以 $1: 3.5$ 的物质的量比, $60{ }^{\circ} \mathrm{C}$ 反应 $6 \mathrm{~h}$, 异腈酸 酯底物几乎都完全转化生成甲基胺硼化物, 即使是空间 位阻非常大的叔丁基和金刚烷取代基，反应也能顺利进 行(Table 5 , Entries 2 6). 令人高兴的是, 当底物为芳香 族异腈酸酯, 异腈酸酯与频哪醇硼烷的物质的量比为 
表 52 催化的异腈酸酯嗍氢化反应

Table 5 Hydroboration of isocyanate catalyzed by 2

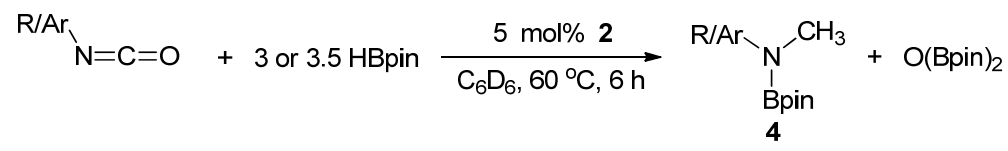

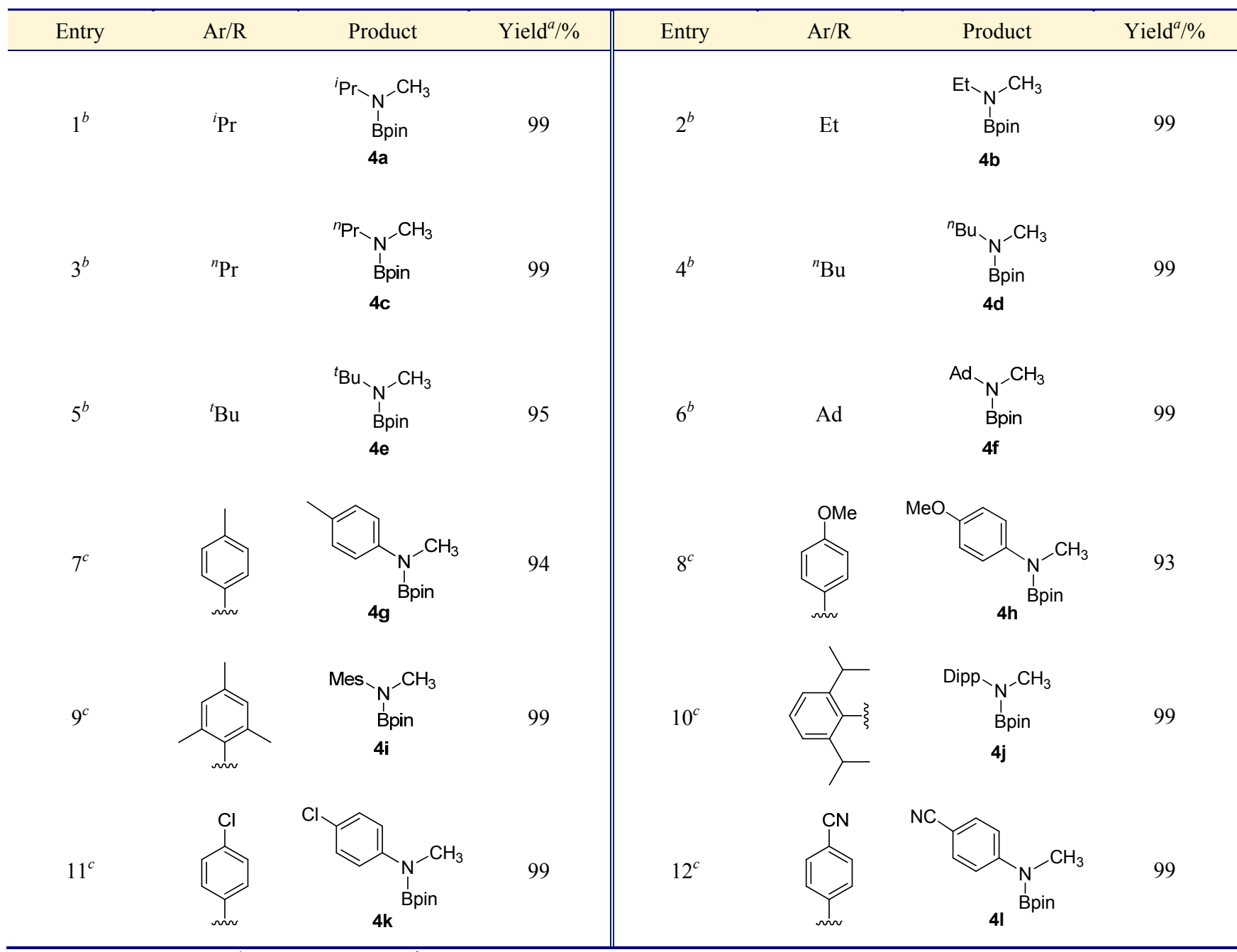

${ }^{a}$ The yield was determined by ${ }^{1} \mathrm{H}$ NMR spectroscopy $;{ }^{b} \mathrm{RNCO} / \mathrm{HBpin}(n: n=1: 3.5) ;{ }^{c} \operatorname{Ar}-\mathrm{NCO} / \mathrm{HBpin}(n: n=1: 3)$.

$1: 3$ 时, 相应的硼氢化在同样的反应条件下也能在较 短时间内获得非常高的产率. 当苯环的对位为甲基或甲 氧基供电子基团时, 产率稍微有所降低(Table 5, Entries $7,8)$ ，而大空间位阻的取代基如 2,4,6-三甲基苯基或 2,6二异丙基苯基时，反应反而非常快，几乎定量反应 (Table 5, Entries 9,10). 这与 Hill等报道的镁催化的芳香 族异腈酸酯硼氢化反应正好相反，当苯环上的取代基空 间位阻越大，产率越低. 例如当 $10 \mathrm{~mol} \%$ 镁化合物催化 2,6-二异丙基苯基异腈酸酯硼氢化反应时, 反应 $24 \mathrm{~h}$, 只获得 50\%产率 ${ }^{[24]}$. 当苯环上的取代基为吸电子基团如 氯基和氰基，在 $5 \mathrm{~mol} \% 2$ 的催化下同样得到 $99 \%$ 产率 (Table 5, Entries 11, 12); 当苯环对位上的取代基为乙酰 基时，反应比较复杂，无法得到相应的目标产物.

总的来说, $\mathrm{Zn}-\mathrm{Li}$ 双金属化合物 2 在异腈酸酯硼氢化
反应上的催化效果比之前报道的镁催化剂高效(Zn-Li: 5 mol\% cat., 93\% 99\%产率; vs. Mg: 10 mol\% cat., 50\% 90\%产率). 最后也尝试了类似结构异硫氰酸苯酯 (PhNCS)的嗍氢化反应, 然而即使与过量的 HBpin (1:5 物质的量比)在 $80{ }^{\circ} \mathrm{C}, 10 \mathrm{~mol} \% 2$ 的催化下也未发生相 应的硼氢化反应.

为了进一步深入理解 Zn-Li 双金属化合物 2 催化的 异腈酸酯硼氢化反应，我们对其反应机理进行了初步探 索. 之前 Fang 和 Hill 等 ${ }^{[24]}$ 报道镁催化的异腈酸酯硼氢 化是分步反应，我们据此也作了初步的定量反应. 以 $\mathrm{C}_{6} \mathrm{D}_{6}$ 为溶剂, $5 \mathrm{~mol} \%$ 催化剂 2 的作用下, ${ }^{i} \mathrm{Pr}-\mathrm{NCO}$ 与 $n$ 等 份 $(n=1,2,3)$ 的 HBpin 分别在室温下反应 $6 \mathrm{~h}$. 通过对反 应混合物的氢谱分析发现，异丙基异腈酸酯与频哪醇硼 烷的物质的量比为 $1: 1$ 或 $1: 2$ 时, 在氢谱化学位移 $\delta$ 
9.02 处有一个明显的甲酰基氢，同时异丙基的次甲基 ( $\mathrm{CH}$, 七重峰)化学位移由 $\delta 3.02$ 转变为 $\delta 4.66$, 因此反 应后主要产物为单硼氢化产物甲酰胺硼化物 ${ }^{i} \mathrm{Pr}-$ $\mathrm{N}$ (Bpin)CHO 以及部分未反应的异丙基异腈酸酯原料 (图 3A 和 3B). 随着频哪醇硼烷的比例增大 $(n=3)$, 原料 异丙基异腈酸酯完全消失, 只有单硼氢化产物甲酰胺砋 化物和过量的频哪醇硼烷(图 3C).

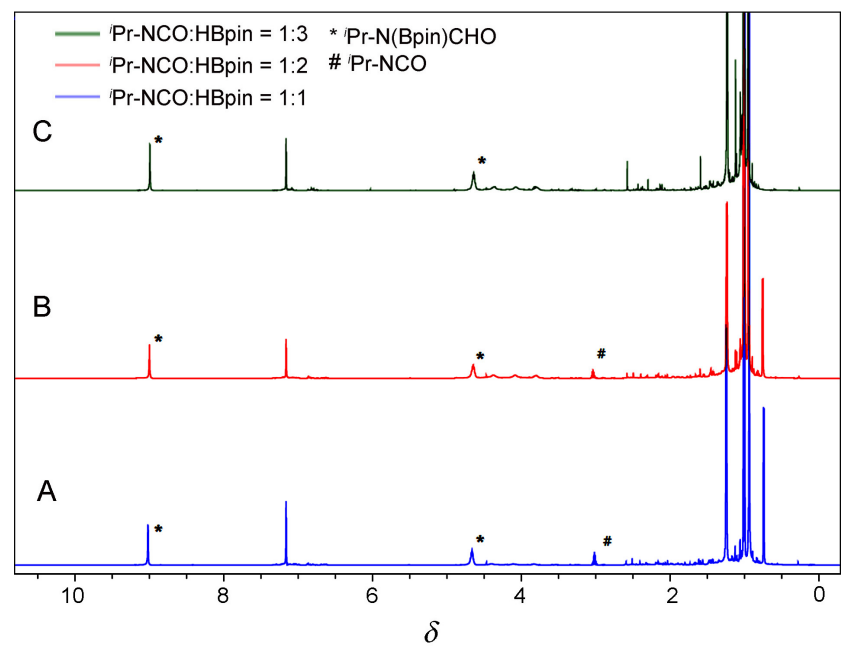

图 $3{ }^{i} \mathrm{Pr}-\mathrm{NCO} / \mathrm{HBpin}$ 的物质的量比分别为 $1: 1$ (A), $1: 2$ (B) 和 $1: 3(\mathrm{C})$ 的 ${ }^{1} \mathrm{H}$ NMR 图 $\left(25{ }^{\circ} \mathrm{C}, 6 \mathrm{~h}\right)$

Figure $3{ }^{1} \mathrm{H}$ NMR spectra of the hydroboration between ${ }^{i}$ Pr-NCO and HBpin in $1: 1$ (A), $1: 2$ (B) and $1: 3$ (C) molar ratio $\left(25{ }^{\circ} \mathrm{C}, 6 \mathrm{~h}\right)$

将反应温度升高至 $60{ }^{\circ} \mathrm{C}$ 反应 $1 \mathrm{~h}$, 当异腈酸酯与频 哪醇嗍烷的物质的量比为 $1: 1$ 时, 与室温时一样, 主要 产物为单硼氢化产物甲酰胺硼化物 ${ }^{i} \mathrm{Pr}-\mathrm{N}$ (Bpin)CHO 以 及部分未反应的异丙基异腈酸酯原料(图 4A). 随着频哪 醇硼烷的比例增大 $(n=2$ 或 3$)$, 原料异丙基异腈酸酯

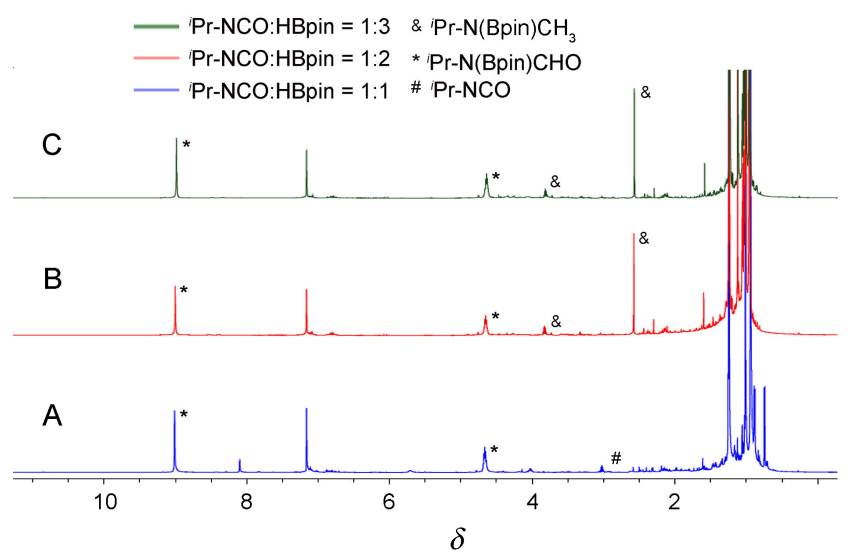

图 $4{ }^{i} \mathrm{Pr}-\mathrm{NCO} / \mathrm{HBpin}$ 的物质的量比分别为 $1: 1$ (A), $1: 2$ (B) 和 $1: 3(\mathrm{C})$ 时的 ${ }^{1} \mathrm{H}$ NMR 图 $\left(60{ }^{\circ} \mathrm{C}, 1 \mathrm{~h}\right)$

Figure $4{ }^{1} \mathrm{H}$ NMR spectra of the hydroboration between ${ }^{i} \mathrm{Pr}$ NCO and HBpin in $1: 1(\mathrm{~A}), 1: 2(\mathrm{~B})$ and $1: 3$ (C) molar ratio $\left(60{ }^{\circ} \mathrm{C}, 1 \mathrm{~h}\right)$
完全消失，除了单硼氢化产物外，同时生成部分完全砋 氢化产物 ${ }^{i} \mathrm{PrN}(\mathrm{Bpin}) \mathrm{CH}_{3}$ (图 $4 \mathrm{~B}$ 和 $4 \mathrm{C}$ ). 令人惊奇的是: 当异腈酸酯与频哪醇硼烷的物质的量比为 $1: 2$ 时，与 Okuda 和 Hill 等 ${ }^{[23-24]}$ 之前的报道不同, 无论室温或者高 温, 都没有发现双嗍氢化产物 ${ }^{i} \mathrm{PrN}(\mathrm{Bpin}) \mathrm{CH}_{2} \mathrm{OBpin}$ 的生 成，只观察到单硼氢化和完全硼氢化的产物(图 3B, 4B). 由于无论室温还是 $60{ }^{\circ} \mathrm{C}$ 都无法观察到相应双嗍氢化产 物 ${ }^{i} \operatorname{PrN}$ (Bpin) $\mathrm{CH}_{2} \mathrm{OBpin}$, 说明该步为整个反应的决速步 骤. 升高温度对第二步反应速率提升明显，而第三步反 应则相对容易进行. 当反应温度升高 $\left(25{ }^{\circ} \mathrm{C}\right.$ vs. $\left.60{ }^{\circ} \mathrm{C}\right)$, 完全硼氢化产物随之增加(图 3C, 4C).

另外，尝试了在室温下 $\mathrm{C}_{6} \mathrm{D}_{6}$ 溶剂中, $\mathrm{Zn}-\mathrm{Li}$ 双金属 化合物 2 与 HBpin 的 1:1 物质的量比反应, 通过氢谱 $\left({ }^{1} \mathrm{H} N M R\right)$ 和嗍谱 $\left({ }^{11} \mathrm{~B} \mathrm{NMR}\right)$ 监测. 随着反应时间的延长, 氢谱中化学位移 $\delta 4.47$ 处逐渐出现一处单峰, 根据已报 道的相关锌氢化物实验数据 $(\delta \quad 4.39 \sim 4.66)^{[28-29]}$, 推测其 应为锌氢化物 [LZnH] 的峰(图 5). 而在嗍谱 $\delta 21.7$ 处出 现的峰为副产物 $\mathrm{O}(\mathrm{Bpin})_{2}$ (图 6). 基于以上数据我们推 测, 2 催化异腈酸酯与频哪醇硼烷的反应中, 实际参与 反应的催化剂可能为锌的氢化物 [LZnH], 而该氢化物 可由 2 与 HBpin 反应生成，同时产生副产物硼醚 $\mathrm{O}(\mathrm{B}-$ pin) $)_{2}$.

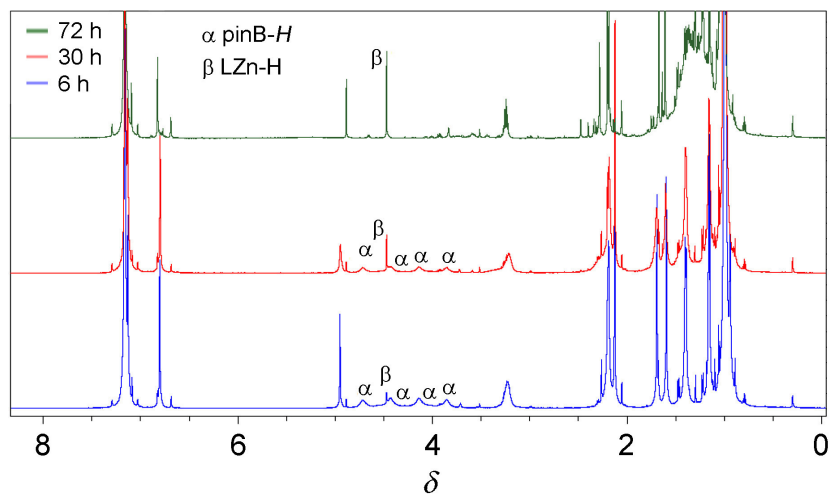

图 $5 \mathrm{HBpin}$ 与 2 的 1: 1 物质的量比反应随时间变化的 ${ }^{1} \mathrm{H}$ NMR 图

Figure $5{ }^{1} \mathrm{H}$ NMR spectra of reaction between 2 and HBpin in $1: 1$ molar ratio with different reaction time

综上所述, Zn-Li 双金属化合物 2 催化的异腈酸酯和 频哪醇硼烷的硼氢化反应为分步反应：化合物 2 先与 HBpin 反应生成锌氢化物, 然后在锌氢化物催化下, 异 腈酸酯先与一等份 HBpin 生成单硼氢化物产物甲酰胺 碍化物, 其随后再与第二等份 HBpin 反应生成双硼氢化 物产物亚甲基胺嗍化物, 最后再与第三等份 HBpin 生成 完全硼氢化产物甲基胺硼化物，同时释放出副产物硼醚 $\mathrm{O}$ (Bpin) $)_{2}$ (Scheme 3). 


$\begin{array}{ll}-72 \mathrm{~h} & \alpha \mathrm{HBpin} \\ -30 \mathrm{~h} & \gamma(\operatorname{pin} B)_{2} \mathrm{O} \\ -6 \mathrm{~h} & \end{array}$

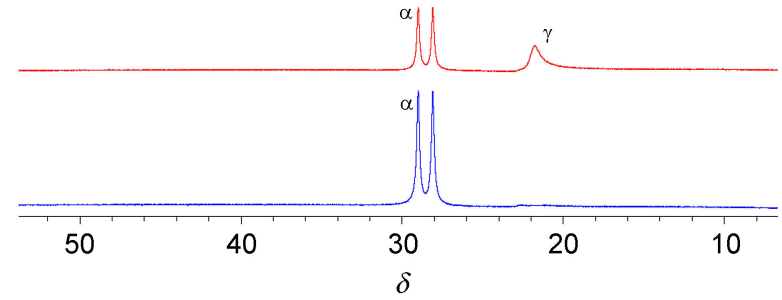

图 $6 \mathrm{HBpin}$ 与 2 的 1: 1 物质的量比的反应随时间变化的 ${ }^{11} \mathrm{~B}$ NMR 图

Figure $6{ }^{11} \mathrm{~B}$ NMR spectra of reaction between 2 and HBpin in $1: 1$ molar ratio with different reaction time
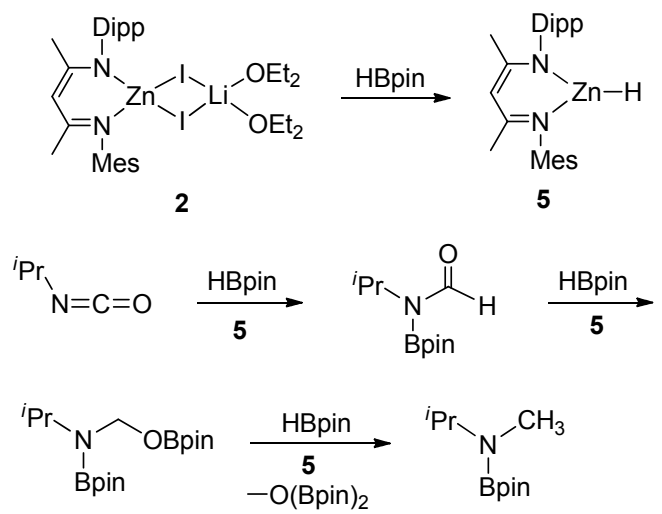

图式 3 异腈酸脂的分步嗍氢化反应

Scheme 3 Stepwise hydroboration of isocyanate

\section{3 结论}

不对称 $\beta$-二亚胺配体被成功应用于锌-锂双金属桥 连碘化物 2 的合成中, 虽然在随后的金属钠还原中没有 得到不对称一价锌化合物, 而只得到单核二聚锌化合 物. 然而锌-锂双金属化合物 2 可以作为高效催化剂应 用于异腈酸酯与频哪醇硼烷的硼氢化反应中, 合成了一 系列带有不同官能团的甲基胺硼化物, 其催化活性要高 于单金属镁催化剂. 通过定量反应, 我们对异腈酸酯硼 氢化的反应机理进行了初步的探索, 认为整个反应是分 步反应。

\section{4 实验部分}

\section{1 试剂与仪器}

所有水、氧敏感反应均使用手套箱及双排管，在高 纯氩气保护下进行. 所有溶剂都在金属钠镜中干燥蒸 馏, 保存于含钠镜的溶剂瓶中. 相关晶体化合物使用 $\mathrm{X}$ 射线单晶衍射进行表征: 挑选合适大小形状的单晶, 使 用 Bruker D8 VENTURE PHOTON 100 在低温 $(130 \mathrm{~K})$ 及 高纯氮保护下搜集相关的单晶衍射数据. 使用
APECK-3 软件程序解析收集好的数据，除氢原子外所 有原子均进行各向异性校正. ${ }^{1} \mathrm{H}$ NMR, ${ }^{11} \mathrm{~B}$ NMR 谱图由 Bruker Avance III $600 \mathrm{MHz}$ 核磁共振仪测定, 化学位移 通过氝代试剂峰进行校正. 配体 1 参照文献[30]方法合 成.

\section{2 锌金属化合物的合成}

不对称 $\beta$-二亚胺锌锂双金属化合物(2): 在一 $78{ }^{\circ} \mathrm{C}$ 下将 ${ }^{n} \mathrm{BuLi}\left(1.66 \mathrm{~mL}, 2.66 \mathrm{mmol}, 1.6 \mathrm{~mol} \cdot \mathrm{L}^{-1}\right.$ in $\left.\mathrm{Et}_{2} \mathrm{O}\right)$ 逐 滴加入配体 1 (1.00 g, $2.66 \mathrm{mmol})$ 的 $\mathrm{Et}_{2} \mathrm{O}(100 \mathrm{~mL})$ 溶液, 逐渐恢复至室温, 摚拌 $6 \mathrm{~h}$. 在 $-78{ }^{\circ} \mathrm{C}$ 下再逐滴加入 $\mathrm{ZnI}_{2}(0.85 \mathrm{~g}, 2.66 \mathrm{mmol})$ 的 $\mathrm{Et}_{2} \mathrm{O}(50 \mathrm{~mL})$ 悬浊液中, 逐渐 恢复至室温，搅拌过夜. 过滤，滤液浓缩至约 $5 \mathrm{~mL}$, $2{ }^{\circ} \mathrm{C}$ 析出无色晶体 2 (1.63 g, 72\%产率). m.p. 182 $184{ }^{\circ} \mathrm{C} ;{ }^{1} \mathrm{H}$ NMR $\left(600 \mathrm{MHz}, \mathrm{C}_{6} \mathrm{D}_{6}\right) \delta: 7.11 \sim 7.16(\mathrm{~m}, 3 \mathrm{H}$, Ar-H), 6.81 (s, 2H, Ar-H), 4.95 (s, 1H, =CH), 3.33 3.30 (m, $10 \mathrm{H}, \mathrm{CH}\left(\mathrm{CH}_{3}\right)_{2}+\mathrm{OCH}_{2} \mathrm{CH}_{3}$ overlap), $2.23(\mathrm{~s}, 6 \mathrm{H}$, $\mathrm{Ar}-\mathrm{CH}_{3}$ ), 2.13 (s, 3H, Ar- $\mathrm{CH}_{3}$ ), 1.70 (s, 3H, $\left.\mathrm{NCCH}_{3}\right), 1.60$ (s, 3H, $\left.\mathrm{NCCH}_{3}\right), 1.42$ (d, $\left.J=6.6 \mathrm{~Hz}, 6 \mathrm{H}, \mathrm{CH}\left(\mathrm{CH}_{3}\right)_{2}\right), 1.17$ (d, $\left.J=6.6 \mathrm{~Hz}, 6 \mathrm{H}, \mathrm{CH}\left(\mathrm{CH}_{3}\right)_{2}\right), 1.07$ (t, $J=7.2 \mathrm{~Hz}, 12 \mathrm{H}$, $\mathrm{OCH}_{2} \mathrm{CH}_{3}$ ).

不对称 $\beta$-二亚胺锌化合物(3): 将化合物 $2(0.5 \mathrm{~g}$, $0.59 \mathrm{mmol}$ )溶于 $50 \mathrm{~mL}$ 甲苯, 置于过量钠镜中, 加入 5 $\mathrm{mL} \mathrm{Et}_{2} \mathrm{O}$, 室温摚拌 $5 \mathrm{~d}$. 过滤, 浓缩至约 $10 \mathrm{~mL}$, 缓慢加 入 $5 \mathrm{~mL}$ 正己烷, $2{ }^{\circ} \mathrm{C}$ 析出少量无色晶体 3 (由于晶体较 少无法进行核磁等表征).

\section{3 异腈酸酯硼氢化一般方法}

将催化剂 2 (0.006 mmol, $5 \mathrm{~mol} \%)$ 加入到异腈酸酯 (0.120 mmol) 和频哪醇硼烷 (0.420 mmol，3.5 equiv.; $0.360 \mathrm{mmol}, 3$ equiv. $)$ 的 $\mathrm{C}_{6} \mathrm{D}_{6}(0.5 \mathrm{~mL})$ 溶液中, $60{ }^{\circ} \mathrm{C}$ 搅拌 $6 \mathrm{~h}$. 反应进程由 ${ }^{1} \mathrm{H} N \mathrm{NMR}$ 和 ${ }^{11} \mathrm{~B}$ NMR 监测.

$\mathrm{N}, \mathrm{N}$-甲基,频哪醇嗍烷一异丙基胺(4a): ${ }^{[24]}{ }^{1} \mathrm{H}$ NMR (600 MHz, $\left.\mathrm{C}_{6} \mathrm{D}_{6}\right) \delta: 3.81$ (sept, ${ }^{3} J=6.6 \mathrm{~Hz}, 1 \mathrm{H}, \mathrm{CHMe}_{2}$ ), $2.56(\mathrm{~s}, 3 \mathrm{H}, \mathrm{NMe}), 1.16(\mathrm{~s}, 12 \mathrm{H}, \mathrm{BOCMe}), 1.03\left(\mathrm{~d},{ }^{3} J=\right.$ $\left.6.8 \mathrm{~Hz}, 6 \mathrm{H}, \mathrm{CHMe}_{2}\right) ;{ }^{11} \mathrm{~B} \mathrm{NMR}\left(193 \mathrm{MHz}, \mathrm{C}_{6} \mathrm{D}_{6}\right) \delta: 24.18$.

$\mathrm{N}, \mathrm{N}$-甲基,频哪醇硼烷一乙基胺(4b): ${ }^{[24]}{ }^{1} \mathrm{H}$ NMR (600 $\left.\mathrm{MHz}, \mathrm{C}_{6} \mathrm{D}_{6}\right) \delta: 3.02\left(\mathrm{q},{ }^{3} \mathrm{~J}=7.2 \mathrm{~Hz}, 2 \mathrm{H}, \mathrm{NCH}_{2} \mathrm{Me}\right.$ ), 2.63 (s, $3 \mathrm{H}, \mathrm{NMe}), 1.12\left(\mathrm{~s}, 12 \mathrm{H}, \mathrm{BOCMe}_{2}\right), 1.11(\mathrm{~s}, 12 \mathrm{H}, \mathrm{BOC}-$ $\left.\mathrm{Me}_{2}\right), 0.99\left(\mathrm{t},{ }^{3} J=7.2 \mathrm{~Hz}, 3 \mathrm{H}, \mathrm{NCH}_{2} \mathrm{Me}\right) ;{ }^{11} \mathrm{~B}$ NMR (193 $\left.\mathrm{MHz}, \mathrm{C}_{6} \mathrm{D}_{6}\right) \delta: 24.24$.

$\mathrm{N}, \mathrm{N}$-甲基,频哪醇硼烷一正丙基胺(4c): ${ }^{[24]}{ }^{1} \mathrm{H} \mathrm{NMR}$ $\left(600 \mathrm{MHz}, \mathrm{C}_{6} \mathrm{D}_{6}\right) \delta: 2.96\left(\mathrm{t},{ }^{3} J=7.2 \mathrm{~Hz}, 2 \mathrm{H}, \mathrm{NCH}_{2}-\right.$ $\mathrm{CH}_{2} \mathrm{Me}$ ), $2.62(\mathrm{~s}, 3 \mathrm{H}, \mathrm{NMe}), 1.38 \sim 1.44\left(\mathrm{~m}, 2 \mathrm{H}, \mathrm{NCH}_{2}-\right.$ $\mathrm{CH}_{2} \mathrm{Me}$ ), 1.12 (s, $12 \mathrm{H}, \mathrm{BOCMe}_{2}$ ), $0.82\left(\mathrm{t},{ }^{3} \mathrm{~J}=7.2 \mathrm{~Hz}, 3 \mathrm{H}\right.$, $\left.\mathrm{NCH}_{2} \mathrm{CH}_{2} \mathrm{Me}\right) ;{ }^{11} \mathrm{~B}$ NMR $\left(193 \mathrm{MHz}, \mathrm{C}_{6} \mathrm{D}_{6}\right) \delta: 24.41$.

$\mathrm{N}, \mathrm{N}$-甲基,频哪醇硼烷一正丁基胺(4d): ${ }^{[24]}{ }^{1} \mathrm{H} \mathrm{NMR}$ 
$\left(600 \mathrm{MHz}, \mathrm{C}_{6} \mathrm{D}_{6}\right) \delta: 3.01\left(\mathrm{t},{ }^{3} J_{\mathrm{HH}}=7.2 \mathrm{~Hz}, 2 \mathrm{H}, \mathrm{NCH}_{2}-\right.$ $\mathrm{CH}_{2} \mathrm{CH}_{2} \mathrm{Me}$ ), $2.64(\mathrm{~s}, 3 \mathrm{H}, \mathrm{NMe}), 1.42 \sim 1.37(\mathrm{~m}, 2 \mathrm{H}$, $\left.\mathrm{NCH}_{2} \mathrm{CH}_{2} \mathrm{CH}_{2} \mathrm{Me}\right), 1.29 \sim 1.23\left(\mathrm{~m}, 2 \mathrm{H}, \mathrm{NCH}_{2} \mathrm{CH}_{2} \mathrm{CH}_{2}-\right.$ $\mathrm{Me}), 1.12\left(\mathrm{~s}, 12 \mathrm{H}, \mathrm{BOCMe}_{2}\right), 0.88\left(\mathrm{t},{ }^{3} J=7.2 \mathrm{~Hz}, 3 \mathrm{H}\right.$, $\mathrm{NCH}_{2} \mathrm{CH}_{2} \mathrm{CH}_{2} \mathrm{Me}$ ); ${ }^{11} \mathrm{~B} \mathrm{NMR}\left(193 \mathrm{MHz}, \mathrm{C}_{6} \mathrm{D}_{6}\right) \delta: 24.36$.

$N, N$-甲基, 频哪醇硼烷一叔丁基胺(4e): ${ }^{[24]}{ }^{1} \mathrm{H} \mathrm{NMR}$ $\left(600 \mathrm{MHz}, \mathrm{C}_{6} \mathrm{D}_{6}\right) \delta: 2.70(\mathrm{~s}, 3 \mathrm{H}, \mathrm{NMe}), 1.29(\mathrm{~s}, 9 \mathrm{H}$, $\mathrm{CMe}_{3}$ ), 1.09 (s, 12H, BOCMe $)$; ${ }^{11} \mathrm{~B}$ NMR (193 MHz, $\left.\mathrm{C}_{6} \mathrm{D}_{6}\right) \delta: 24.35$.

$N, N$-甲基,频哪醇硼烷-金刚烷基胺(4f): ${ }^{[24]}{ }^{1} \mathrm{H} \mathrm{NMR}$ $\left(600 \mathrm{MHz}, \mathrm{C}_{6} \mathrm{D}_{6}\right) \delta: 2.75$ (s, 3H, NMe), 2.01 1.57 (m, 15H, Ad-H), 1.11 (s, 12H, BOCMe $),{ }^{11} \mathrm{~B}$ NMR (193 MHz, $\left.\mathrm{C}_{6} \mathrm{D}_{6}\right) \delta: 24.36$.

$N, N$-甲基,频哪醇硼烷-对甲苯基胺(4g): ${ }^{[24]}{ }^{1} \mathrm{H} \mathrm{NMR}$ $\left(600 \mathrm{MHz}, \mathrm{C}_{6} \mathrm{D}_{6}\right) \delta: 7.40 \sim 7.39$ (m, 2H, Ar-H), 7.05 7.03 (m, 2H, Ar-H), 3.04 (s, 3H, NMe), 2.14 (s, 3H, ArMe), $1.08\left(\mathrm{~s}, 12 \mathrm{H}, \mathrm{BOCMe}_{2}\right) ;{ }^{11} \mathrm{~B}$ NMR $\left(193 \mathrm{MHz}, \mathrm{C}_{6} \mathrm{D}_{6}\right) \delta$ : 24.68 .

$N, N$-甲基, 频哪醇嗍烷-对甲氧基苯基胺(4h): ${ }^{[24]}{ }^{1} \mathrm{H}$ NMR (600 MHz, $\left.\mathrm{C}_{6} \mathrm{D}_{6}\right) \delta: 7.35 \sim 7.34(\mathrm{~m}, 2 \mathrm{H}, \mathrm{Ar}-\mathrm{H})$, $6.81 \sim 6.82(\mathrm{~m}, 2 \mathrm{H}, \mathrm{Ar}-\mathrm{H}), 3.37$ (s, 3H, NMe), 3.04 (s, 3H, ArOMe), 1.09 (s, 12H, BOCMe $)$; ${ }^{11} \mathrm{~B}$ NMR (193 MHz, $\left.\mathrm{C}_{6} \mathrm{D}_{6}\right) \delta: 24.61$.

$N, N$-甲基,频哪醇硼烷-2,4,6-三甲基苯基胺(4i): ${ }^{[24]}$ ${ }^{1} \mathrm{H}$ NMR (600 MHz, $\left.\mathrm{C}_{6} \mathrm{D}_{6}\right) \delta: 6.77$ (s, 2H, Ar-H), 2.86 (s, $3 \mathrm{H}, \mathrm{NMe}$ ), 2.26 (s, 6H, Ar-Me), 2.10 (s, 3H, Ar-Me), 1.00 $\left(\mathrm{s}, 12 \mathrm{H}, \mathrm{BOCMe}_{2}\right) ;{ }^{11} \mathrm{~B}$ NMR $\left(193 \mathrm{MHz}, \mathrm{C}_{6} \mathrm{D}_{6}\right) \delta: 24.30$.

$N, N$-甲基,频哪醇嗍烷-2,6-二异丙基苯基胺 (4j j): ${ }^{[24]}$ ${ }^{1} \mathrm{H}$ NMR (600 MHz, $\left.\mathrm{C}_{6} \mathrm{D}_{6}\right) \delta: 7.09 \sim 7.08$ (m, 3H, Ar-H), $\delta$ 3.35 (sept, ${ }^{3} J=7.2 \mathrm{~Hz}, 2 \mathrm{H}, \mathrm{Ar}-\mathrm{CHMe}_{2}$ ), 2.90 (s, 3H, NMe), $2.31 \sim 1.22\left(\mathrm{~m}, 12 \mathrm{H}, \mathrm{Ar}-\mathrm{CHMe}_{2}\right), 1.18$ (s, 12H, BOCMe $)_{2}$; ${ }^{11} \mathrm{~B}$ NMR (193 MHz, $\left.\mathrm{C}_{6} \mathrm{D}_{6}\right) \delta: 24.20$.

$N, N$-甲基, 频哪醇硼烷-对氯苯基胺(4k): ${ }^{[24] ~}{ }^{1} \mathrm{H}$ NMR $\left(600 \mathrm{MHz}, \mathrm{C}_{6} \mathrm{D}_{6}\right) \delta: 7.22 \sim 7.21(\mathrm{~m}, 2 \mathrm{H}, \mathrm{Ar}-\mathrm{H}), 7.16 \sim 7.15$ $(\mathrm{m}, 2 \mathrm{H}, \mathrm{Ar}-\mathrm{H}), 2.87 \sim 2.86(\mathrm{~m}, 3 \mathrm{H}, \mathrm{NMe}), 1.06 \sim 1.05(\mathrm{~m}$, $\left.12 \mathrm{H}, \mathrm{BOCMe}_{2}\right) ;{ }^{11} \mathrm{~B}$ NMR (193 MHz, $\mathrm{C}_{6} \mathrm{D}_{6}$ ) $\delta: 24.97$.

$N, N$-甲基,频哪醇嗍烷一对氰基苯基胺 (4l): ${ }^{[24]}{ }^{1} \mathrm{H}$ NMR (600 MHz, $\left.\mathrm{C}_{6} \mathrm{D}_{6}\right) \delta: 7.16 \sim 7.15$ (m, 4H, Ar-H), 2.73 $(\mathrm{s}, 3 \mathrm{H}, \mathrm{NMe}), 1.03\left(\mathrm{~s}, 12 \mathrm{H}, \mathrm{BOCMe}_{2}\right) ;{ }^{11} \mathrm{~B} \mathrm{NMR}$ (193 $\left.\mathrm{MHz}, \mathrm{C}_{6} \mathrm{D}_{6}\right) \delta: 24.64$.

辅助材料(Supporting Information) 嗍氢化产物 $4 \mathrm{a} \sim$
41 的核磁共振氢谱和硼谱谱图. 这些材料可以从 http:// sioc-journal.cn/上免费下载.

\section{References}

[1] Bravo-Zhivotovskii, D.; Yuzefovich, M.; Bendikov, M.; Klinkhammer, K.; Apeloig, Y. Angew. Chem., Int. Ed. 1999, 38, 1100.

[2] Resa, I.; Carmona, E.; Gutierrez-Puebla, E.; Monge, A. Science 2004, 305, 1136.

[3] Del Río, D.; Galindo, A.; Resa, I.; Carmona, E. Angew. Chem., Int. Ed. 2005, 44, 1244.

[4] Li, T.; Schulz, S.; Roesky, P. W. Chem. Soc. Rev. 2012, 41, 3759.

[5] Cao, C.; Shi, Y.; Xu, H.; Zhao, B. Coord. Chem. Rev. 2018, 365, 122.

[6] Chen, M.; Jiang, S.; Maron, L.; Xu, X. Dalton Trans. 201948 , 1931.

[7] Freitag, K.; Banh, H.; Ganesamoorthy, C.; Gemel, C.; Seidel, R. W.; Fischer, R. A. Dalton Trans. 2013, 42, 10540.

[8] Bollermann, T.; Gemel, C.; Fischer, R. A. Coord. Chem. Rev. 2012, 256, 537.

[9] Lühl, A.; Pada, N. H.; Blechert, S.; Roesky, P. W. Chem. Commun. 2011, 47,8280 .

[10] Lühl, A.; Hartenstein, L.; Blechert, S.; Roesky, P. W. Organometallics 2012, 31, 7109.

[11] Chong, C. C.; Kinjo, R. ACS Catal. 2015, 5, 3238.

[12] Yoshida, H. ACS Catal. 2016, 6, 1799.

[13] Luo, M.; Zang, S.; Yao, W.; Zheng, J.; Ma, M. Sci. Sin. Chim. 2020, 50,639 .

[14] Zhou, N.; Yuan, X.; Zhao, Y.; Xie, J.; Zhu, C. Angew. Chem., Int. Ed. 2018, 57, 3990.

[15] Shimoi, M.; Watanabe, T.; Maeda, K.; Curran, D. P.; Taniguchi, T. Angew. Chem., Int. Ed. 2018, 57, 9485.

[16] Ren, S.; Zhang, F.; Xu, A.; Yang, Y.; Zheng, M.; Zhou, X.; Fu, Y.; Wang, Y. Nat. Commun. 2019, 10, 1934.

[17] Huang, Y.; Wang, J.; Zheng, W.; Zhang, F.; Yu, Y.; Zheng, M.; Zhou X.; Wang Y. Chem. Commun. 2019, 55, 11904.

[18] Li, T.; Zhang, J.; Cui, C. Chin. J. Chem. 2019, 37, 679.

[19] Xu, X.; Kang, Z.; Yan, D.; Xue, M. Chin. J. Chem. 2019, 37, 1142.

[20] Liu, X.; Zhu, Q.; Chen, D.; Wang, L.; Jin, L.; Liu, C. Angew. Chem., Int. Ed. 2020, 59, 2745.

[21] Tamang, S. R.; Singh, A.; Bedi, D.; Bazkiaei, A. R.; Warner, A. A.; Glogau, K.; Mcdonald, C.; Unruh, D. K.; Findlater, M. Nat. Catal. 2020, 3,154 .

[22] Mcquilken, A. C.; Dao, Q. M.; Cardenas, A. J. P.; Bertke, J. A.; Grimme, S.; Warren, T. H. Angew. Chem. Int. Ed. 2016, 55, 14335.

[23] Mukherjee, D.; Shirase, S.; Spaniol, T. P.; Mashima, K.; Okuda, J. Chem. Commun. 2016, 52, 13155.

[24] Yang, Y.; Anker, M. D.; Fang, J.; Mahon, M. F.; Maron, L.; Weetman, C.; Hill, M. S. Chem. Sci. 2017, 8, 3529.

[25] Solé, C.; Fernández, E. Angew. Chem., Int. Ed. 2013, 52, 11351.

[26] Li, J.; Luo, M.; Sheng, X.; Hua, H.; Yao, W.; Pullarkat, S. A.; Xu, L.; Ma, M. Org. Chem. Front 2018, 5, 3538.

[27] Prust, J.; Most, K.; Müller, I.; Stasch, A.; Roesky, H. W.; Usón, I. Eur. J. Inorg. Chem. 2001, 1613.

[28] Spielmann, J.; Piesik, D.; Wittkamp, B.; Jansen, G.; Harder, S. Chem. Commun. 2009, 23, 3455.

[29] Lummis, P. A.; Momeni, M. R.; Lui, M. W.; Mcdonald, R.; Ferguson, M. J.; Miskolzie, M.; Brown, A.; Rivard, E. Angew. Chem., Int. Ed. 2014, 53, 9347.

[30] Gong, S.; Ma, H. Dalton Trans. 2008, 25, 3345. 